\title{
Comparison of Two Simulation-based Methods for Modeling Plant Growth
}

\author{
Douglas A. Hopper ${ }^{1}$ \\ Department of Horticulture, Colorado State University, Fort Collins, CO 80523 \\ P. Allen Hammer ${ }^{2}$ \\ Department of Horticulture, Purdue University, West Lafayette, IN 47907 \\ James R. Wilson ${ }^{2}$ \\ Department of Industrial Engineering, North Carolina State University, Raleigh, NC 27695-7906
}

\begin{abstract}
To predict plant growth as a function of time and environmental conditions, plant scientists frequently use empirical mathematical models. In many applications, a realistic model of plant growth is too complex to be manipulated directly by the user; instead the model must be implemented in a computer simulation that can be used as a vehicle for largescale experimentation. Construction of a computer simulation of plant growth requires that the user identify the primary growth responses (characteristics) of interest and formulate equations that accurately represent the relationship between those responses and relevant environmental variables as well as elapsed time. The accuracy of predictions of plant growth will depend not only on the appropriateness of the mathematical form of the prediction equations, but also on the accuracy with which the initial conditions and the equation coefficients are estimated. In this paper we compare the prediction accuracy of two closely related methods for simulating the growth of 'Royalty' roses (Rosa hybrida L.). Also, we explore why one of these methods, an additive approach, generally yields substantially more accurate predictions of nine primary plant growth characteristics at flowering than does a multiplicative approach. The main findings of this paper should be applicable to the development of accurate simulation-based growth models for other plants.
\end{abstract}

We divide our presentation into five sections. In the rest of this first section, we elaborate the fundamental problem that motivated this paper. In the second section we detail a method for modeling the growth of 'Royalty' roses that has been implemented in the simulation program ROSESIM (Hopper et al., 1994). In the third section we describe an alternative method for simulating plant growth, and we explain the potential theoretical problems with this method. In the fourth section we present an experimental comparison of the

Received for publication 11 Oct. 1994. Accepted for publication 28 Apr. 1995. Funding was provided by Colorado Agricultural Experiment Station (project 747) and jointly with the Purdue Univ. Agricultural Experiment Station. The cost of publishing this paper was defrayed in part by the payment of page charges. Under postal regulations, this paper therefore must be hereby marked advertisement solely to indicate this fact.

${ }^{1}$ Assistant Professor.

${ }^{2}$ Professor. prediction accuracy of ROSESIM vs. the alternative simulation method. The main conclusions of the current work and recommendations for future work are summarized in the fifth section.

Although our experimental comparison of methods for simulating plant growth is limited to the situation in which all relevant environmental conditions are maintained at constant levels, systematic variation of these levels throughout the region of interest in the design space ensures that some general conclusions can be drawn about the relative performance of the selected simulation methods. There are an almost limitless number of alternative methods for simulating plant growth, but we have confined our discussion to two methods that seemed to be particularly appropriate in relation to much of our previous work, and that we have investigated in depth. In particular, we focus much of the discussion on the mathematical and statistical basis for ROSESIM. The operation of ROSESIM consists of two phases. The preliminary growth phase begins at the time of hard-pinching and ends 10 days later, at which time nine plant growth characteristics (stem length and the following fresh and dry weights: stem, leaf, flower, and total) are predicted by quadratic functions of photosynthetic photon flux (PPF), day temperature (DT), and night temperature (NT). The main growth phase then follows and ends at the time of flowering (38 to 75 days after pinching); during this phase, daily growth increments of all nine characteristics are predicted by thirddegree polynomial functions of PPF, DT, NT, and $t$, the elapsed time since hard-pinching.

Our primary objective is to compare the prediction accuracy of ROSESIM at flowering to that of an alternative simulation model for the main growth phase in which daily increments of the logarithmically transformed plant growth characteristics are predicted by third-degree polynomial functions of PPF, DT, $\mathrm{NT}$, and $t$. Daily increments of each log-transformed response are accumulated by summing over the main growth phase, and then exponentiated to recover the original (untransformed) plant growth characteristic at flowering. We present experimental evidence that, in the alternative simulation model of the main growth phase, the prediction error for the initial condition of each plant growth characteristic (i.e., the estimation error for each characteristic at the end of the preliminary growth phase) often translates into an unacceptably large prediction error for the final value of that characteristic at flowering. We conclude that the ROSESIM growth model is more robust against prediction errors in both the preliminary and main growth phases; thus, ROSESIM can produce substantially more accurate predictions of all plant growth characteristics at flowering.

\section{ROSESIM GROWTH MODEL}

We begin by defining the notation that is used throughout the rest of this paper. We take the time origin (i.e., $t=0$ ) to be the time of hard-pinching; and we let $t_{\text {beg }}$ and $t_{\text {end }}$, respectively, denote the beginning and ending times for the main growth phase. In ROSESIM, we take $t_{\text {beg }}=10$ days; and $t_{\text {end }}$ (the time of flowering) is approximated by a quadratic function of PPF, DT, and NT as described in Hopper and Hammer (1991). We let $z(t)$ denote a plant growth characteristic of interest at time $t, 0 \leq$ $t \leq t_{\text {end }}$; and for concreteness throughout this discussion, we assume that $z(t)$ is the total plant dry matter at time $t$.

At the end of the preliminary growth phase (i.e., when $t=t_{\text {beg }}$ ), the plant growth characteristic $z(t)$ is predicted as a quadratic function of the independent variables $X_{\mathrm{PPF}}, X_{\mathrm{DT}}$, and $X_{\mathrm{NT}}$, which are coded versions of the constant environmental factors PPF, DT, and NT, respectively, as described in Hopper et al. (1994). Specifically, $z\left(t_{\text {beg }}\right)$ is assumed to have the form

$$
\begin{gathered}
z\left(t_{\mathrm{beg}}\right)=g(\mathbf{X} ; \mathbf{d}) \equiv d_{0}+d_{1} X_{\mathrm{PPF}}+d_{2} X_{\mathrm{DT}}+d_{3} X_{\mathrm{NT}}+ \\
d_{11} X_{\mathrm{PPF}}^{2}+d_{22} X_{\mathrm{DT}}^{2}+d_{33} X_{\mathrm{NT}}^{2}+d_{12} X_{\mathrm{PPF}} X_{\mathrm{DT}}+ \\
d_{13} X_{\mathrm{PPF}} X_{\mathrm{NT}}+d_{23} X_{\mathrm{DT}} X_{\mathrm{NT}},
\end{gathered}
$$

where

$\mathbf{X} \equiv\left[X_{\mathrm{PPF}}, X_{\mathrm{DT}}, X_{\mathrm{NT}}\right]$ and $\mathbf{d} \equiv\left[d_{0}, d_{1}, \ldots, d_{23}\right]$ [2]

denote the vector of coded independent variables and the vector of regression coefficients in the linear regression model [1], respectively, for $z\left(t_{\text {beg }}\right)$. As described in Hopper et al. (1994), the ordinary least squares estimator $\widehat{\boldsymbol{d}} \equiv\left[\hat{d}_{0}, \hat{d}_{1}, \ldots, \hat{d}_{23}\right]$ of $\mathbf{d}$ is computed from plant responses observed in a prior growth chamber experiment so that $\hat{\mathbf{d}}$ can be used to obtain the prediction

$\hat{z}\left(t_{\text {beg }}\right)=g(\mathbf{X} ; \hat{\mathbf{d}})$

for the initial value of the characteristic in the main growth phase. 
Throughout the main growth phase, the time step $\Delta t=1$ day is used; and the increment of growth on day $t$,

$\Delta z(t) \equiv z(t)-z(t-\Delta t)=z(t)-z(t-1)$, for $t=t_{\mathrm{beg}}+1, t_{\mathrm{beg}}+2, \ldots, t_{\mathrm{end}}$,

is assumed to depend on $X_{\mathrm{PPF}}, X_{\mathrm{DT}}, X_{\mathrm{NT}}$, and the coded time

$X_{t}=t / 10$

according to the third-degree polynomial function

$\Delta z(t)=f(t, \mathbf{X} ; \mathbf{b}) \equiv b_{0}+b_{t} X_{t}+b_{t t} X_{\mathrm{t}}^{2}+b_{1} X_{\mathrm{PPF}}$

$+b_{2} X_{\mathrm{DT}}+b_{3} X_{\mathrm{NT}}+b_{11} X_{\mathrm{PPF}}^{2}+b_{22} X_{\mathrm{DT}}^{2}+b_{33} X_{\mathrm{NT}}^{2}$

$+b_{12} X_{\mathrm{PPF}} X_{\mathrm{DT}}+b_{13} X_{\mathrm{PPF}} X_{\mathrm{NT}}+b_{23} X_{\mathrm{DT}} X_{\mathrm{NT}}$

$+b_{t 1} X_{t} X_{\mathrm{PPF}}+b_{t 2} X_{t} X_{\mathrm{DT}}+b_{t 3} X_{t} X_{\mathrm{NT}}+b_{t 11} X_{t} X_{\mathrm{PPF}}^{2}$

$+b_{t 22} X_{t} X_{\mathrm{DT}}^{2}+b_{t 33} X_{\mathrm{t}} X_{\mathrm{NT}}^{2}+b_{t 12} X_{t} X_{\mathrm{PPF}} X_{\mathrm{DT}}$

$+b_{t 13} X_{t} X_{\mathrm{PPF}} X_{\mathrm{NT}}+b_{t 23} X_{t} X_{\mathrm{DT}} X_{\mathrm{NT}}$,

where $\mathbf{X}$ is given by Eq. [2] and

$\mathbf{b} \equiv\left[b_{0}, b_{t}, \ldots, b_{t 23}\right]$

denotes the vector of regression coefficients in the linear regression model [6] for $\Delta z(t), t=t_{\mathrm{beg}}$ $+1, t_{\text {beg }}+2, \ldots, t_{\text {end }}$. Thus, the final value of the selected plant growth characteristic at flowering is

$z\left(t_{\text {end }}\right)=z\left(t_{\text {beg }}\right)+\left[\sum_{t=t_{\text {beg }}+1}^{t_{\text {end }}} \Delta z(t)\right]=$
$g(\mathbf{X} ; \mathbf{d})+\left[\sum_{t=t_{\text {beg }}+1}^{t_{\text {end }}} f(t, \mathbf{X} ; \mathbf{b})\right]$.

As described in Hopper et al. (1994), the ordinary least squares estimator $\hat{\boldsymbol{b}} \equiv\left[\hat{b}_{0}, \hat{b}_{t}, \ldots, \hat{b}_{t 23}\right]$ of $\mathbf{b}$ is computed from estimates of the daily increments of each growth response in a prior greenhouse experiment so that $\widehat{\mathbf{d}}$ and $\widehat{\mathbf{b}}$ can be used to obtain the prediction

$\hat{z}\left(t_{\text {end }}\right)=g(\mathbf{X} ; \widehat{\mathbf{d}})+\left[\sum_{t=t_{\text {beg }}+1}^{t_{\text {end }}} f(t, \mathbf{X} ; \widehat{\mathbf{b}})\right]$

of the plant growth characteristic at flowering.

\section{ALTERNATIVE GROWTH MODEL}

In the alternative simulation model for the growth of 'Royalty' roses, we also use Eqs. [1] and [3] to represent the preliminary growth phase; however, in the main growth phase, we work with the logarithmically transformed characteristic

$y(t) \equiv \log _{e}[z(t)], t=t_{\text {beg }}, t_{\text {beg }}+1, \ldots, t_{\text {end }}$.

The logarithmic transformation [10] is often used as a means of obtaining normally distributed observations with constant variance, which is a basic assumption when fitting a classical growth model to the responses of growing plants (Draper and Smith, 1981; Nelder, 1961). Ensuring the validity of this basic assumption is our main motivation for developing an alternative to ROSESIM. As in the ROSESIM model, we work with difference equations based on the time step $\Delta t=1$ day; and in terms of the coded time $X_{t}$ defined by Eq. [5] and the vector $\mathbf{X}$ of coded independent variables defined by Eq. [2], we assume that the daily growth increment has the general functional form

$\Delta y(t)=\varphi(t, \mathbf{X} ; \boldsymbol{\beta}) \equiv \beta_{0}+\beta_{t} X_{t}+\beta_{t t} X_{t}^{2}+\beta_{1} X_{\mathrm{PPF}}$ $+\beta_{2} X_{\mathrm{DT}}+\beta_{3} X_{\mathrm{NT}}+\beta_{11} X_{\mathrm{PPF}}^{2}+\beta_{22} X_{\mathrm{DT}}^{2}+\beta_{33} X_{\mathrm{NT}}^{2}$ $+\beta_{12} X_{\mathrm{PPF}} X_{\mathrm{DT}}+\beta_{13} X_{\mathrm{PPF}} X_{\mathrm{NT}}+\beta_{23} X_{\mathrm{DT}} X_{\mathrm{NT}}$

$+\beta_{t 1} X_{t} X_{\mathrm{PPF}}+\beta_{t 2} X_{t} X_{\mathrm{DT}}+\beta_{t 3} X_{t} X_{\mathrm{NT}}+\beta_{t 11} X_{t} X_{\mathrm{PPF}}^{2}$

$+\beta_{t 22} X_{t} X_{\mathrm{DT}}^{2}+\beta_{t 33} X_{t} X_{\mathrm{NT}}^{2}+\beta_{t 12} X_{t} X_{\mathrm{PPF}} X_{\mathrm{DT}}$

$+\beta_{t 13} X_{t} X_{\mathrm{PPF}} X_{\mathrm{NT}}+\beta_{t 23} X_{t} X_{\mathrm{DT}} X_{\mathrm{NT}}$,

where

$\boldsymbol{\beta} \equiv\left[\beta_{0}, \beta_{t}, \ldots, \beta_{t 23}\right]$

denotes the vector of regression coefficients in the linear regression model [11] for $\Delta y(t), t=$ $t_{\text {beg }}+1, t_{\text {beg }}+2, \ldots, t_{\text {end }}$.

Combining Eq. [1] for the original (untransformed) plant growth characteristic at the beginning of the main growth phase and the difference Eq. [11] for daily increments of the log-transformed characteristic within the main growth phase, we see that the final value of the original (untransformed) characteristic at flowering is

$z\left(t_{\text {end }}\right)=\exp \left[y\left(t_{\text {end }}\right)\right]=\exp \left[y\left(t_{\text {beg }}\right)+\sum_{t=t_{\text {beg }}+1}^{t_{\text {end }}} \Delta y(t)\right]=$

$g(\mathbf{X} ; \mathbf{d}) \cdot \exp \left[\sum_{t=t_{\text {beg }}+1}^{t_{\text {end }}} \varphi(t, \mathbf{X} ; \boldsymbol{\beta})\right]$

Using the ordinary least squares estimates $\widehat{\mathbf{d}}$ and $\widehat{\boldsymbol{\beta}}$ for $\mathbf{d}$ and $\boldsymbol{\beta}$, respectively, as computed from the same prior growth chamber experiment that was used to estimate the parameters of ROSESIM, we obtain the alternative prediction

$\tilde{z}\left(t_{\text {end }}\right)=\hat{z}\left(t_{\mathrm{beg}}\right) \cdot \exp \left[\sum_{t=t_{\mathrm{beg}}+1}^{t_{\text {end }}} \Delta \hat{y}(t)\right]=$

$g(\mathbf{X} ; \widehat{\mathbf{d}}) \cdot \exp \left[\sum_{t=t_{\text {beg }}+1}^{t_{\text {end }}} \varphi(t, \mathbf{X} ; \widehat{\boldsymbol{\beta}})\right]$

of the selected plant growth characteristic at flowering.

Inspection of Eq. [14] reveals the key property of the alternative model for the main growth phase: the predicted final value $\tilde{z}\left(t_{\text {end }}\right)$ is directly proportional to the predicted initial value $\hat{z}\left(t_{\text {beg }}\right)$. If the multiplier

$\exp \left[\sum_{t=t_{\text {beg }}+1}^{t_{\text {end }}} \varphi(t, \mathbf{X} ; \hat{\boldsymbol{\beta}})\right]$

is large relative to $\hat{z}\left(t_{\mathrm{beg}}\right)$, then any error in the initial prediction $\hat{z}\left(t_{\text {beg }}\right)$ will be magnified excessively in the final prediction $\tilde{z}\left(t_{\text {end }}\right)$. If the multiplier [15] is large relative to $\hat{z}\left(t_{\text {beg }}\right)$, then so is the additive term

$\left[\sum_{t=t_{\text {beg }}+1}^{t_{\text {end }}} f(t, \mathbf{X} ; \widehat{\mathbf{b}})\right]$

in Eq. [9]; but in the ROSESIM model of the main growth phase, the purely additive form of Eq. [9] ensures that in this situation, any error in the initial prediction $\hat{z}\left(t_{\text {beg }}\right)$ will have little effect on the final prediction $\hat{z}\left(t_{\text {end }}\right)$. This phenomenon is illustrated in the experimental comparison of the two growth models that is presented next.

\section{COMPARISON OF GROWTH MODELS}

In this section, we illustrate 1) the robustness of ROSESIM against errors in the initial prediction, $\hat{z}\left(t_{\text {beg }}\right)$, and 2$)$ the extreme sensitivity of the alternative growth model to this initial prediction error. Our results are extracted from a larger experiment described in Hopper et al. (1994) in which 15 combinations of PPF, DT, and NT were selected according to a rotatable central composite design to represent commercial greenhouse conditions during the winter and spring in the midwestern United States. Table 1 displays each treatment combination that was used for comparing the growth models together with the corresponding initial prediction $\hat{z}\left(t_{\text {beg }}\right)$ for total plant dry matter. Over the selected treatment combinations, $\hat{z}\left(t_{\text {beg }}\right)$ ranges from 0.00359 to $0.0780 \mathrm{~g}$; thus, the minimal initial prediction differs from the maximal initial prediction by a factor of 21.7 .

For each treatment combination defined by constant environmental conditions PPF, DT, and NT maintained during the main growth phase, Table 2 displays the treatment means at flowering together with the corresponding predictions, $\tilde{z}\left(t_{\text {end }}\right)$ and $\hat{z}\left(t_{\text {end }}\right)$, that were obtained by the alternative growth model and ROSESIM, respectively. For each treatment combination, the alternative model of the main growth phase yielded a final prediction, $\tilde{z}\left(t_{\text {end }}\right)$, that differed from ROSESIM's final prediction, $\hat{z}\left(t_{\text {end }}\right)$, by multiplicative factors ranging up to 21.7 (i.e., 24.94/1.15) (Table 2). In other words, the final prediction, $\tilde{z}\left(t_{\text {end }}\right)$, was more strongly influenced by the initial prediction, $\hat{z}\left(t_{\text {beg }}\right)$, (and thus by the environmental conditions in the preliminary growth phase) than by the environmental conditions in the main growth phase. From both a statistical and a biological point of view, this behavior of $\tilde{z}\left(t_{\text {end }}\right)$ is unacceptable and unrepresentative of the measured plant growth, especially for plants such as roses. By contrast, the final prediction, $\hat{z}\left(t_{\text {end }}\right)$, based on ROSESIM depends primarily on environmental conditions during the main growth phase rather than the initial prediction, $\hat{z}\left(t_{\text {beg }}\right)$.

Although extremely unfavorable environmental conditions may limit growth early on, possibly stunting growth from which the plant 
Table 1. Constant environmental conditions maintained during the preliminary growth phase, and the initial prediction, $\hat{z}\left(t_{\text {beg }}\right)$, for the total dry matter at the beginning of the main growth phase. ${ }^{2}$

\begin{tabular}{|c|c|c|c|c|c|c|c|c|}
\hline \multirow[b]{2}{*}{ Treatment } & \multicolumn{5}{|c|}{ Environmental factors ${ }^{y}$} & \multirow{2}{*}{\multicolumn{2}{|c|}{$\begin{array}{l}\hat{z}\left(t_{\mathrm{beg}}\right) \\
(\mathrm{g})\end{array}$}} & \multirow{2}{*}{$\begin{array}{c}\hat{z}\left(t_{\text {beg }}\right) \\
\text { value over } \\
\text { range }\end{array}$} \\
\hline & \multicolumn{3}{|c|}{$\begin{array}{c}\text { PPF } \\
\left(\mu \mathrm{mol} \cdot \mathrm{m}^{-2} \cdot \mathrm{s}^{-1}\right)\end{array}$} & $\begin{array}{l}\text { DT } \\
\left({ }^{\circ} \mathrm{C}\right)\end{array}$ & $\begin{array}{l}\mathrm{NT} \\
\left({ }^{\circ} \mathrm{C}\right)\end{array}$ & & & \\
\hline 2 & \multicolumn{2}{|c|}{100} & & 17 & 14 & \multicolumn{2}{|c|}{0.003588} & $\overline{\text { Minimum }}$ \\
\hline 6 & \multicolumn{2}{|c|}{175} & & 20 & 17 & \multicolumn{2}{|c|}{0.02134} & Mean \\
\hline$--^{x}$ & \multicolumn{2}{|c|}{300} & & 25 & 22 & \multicolumn{2}{|c|}{0.07798} & Maximum \\
\hline \multicolumn{9}{|c|}{$\begin{array}{l}{ }^{2} \text { The preliminary growth phase ends and the main growth phase begins at time } t_{\text {beg }}=10 \text { days after hard- } \\
\text { pinching. Treatment numbers relate directly to those in Hopper et al. }(1994) \text {. } \\
\text { yPPF = photosynthetic photon flux; DT = day temperature; NT = night temperature. } \\
\text { 'Values in this row represent the maximum for each environmental condition on which ROSESIM was } \\
\text { based, although no actual single treatment combination used all these specific values. }\end{array}$} \\
\hline & \multirow{2}{*}{\multicolumn{3}{|c|}{$\begin{array}{c}\text { Main growth- } \\
\text { phase environment }{ }^{z}\end{array}$}} & \multirow{3}{*}{\multicolumn{2}{|c|}{$\begin{array}{c}\text { Initial } \\
\text { prediction } \\
\hat{z}\left(t_{\text {beg }}\right) \\
(\mathrm{g})\end{array}$}} & \multicolumn{2}{|c|}{ Final prediction } & \multirow{3}{*}{$\begin{array}{c}\text { Treatment } \\
\text { mean }^{y} \\
(\mathrm{~g})\end{array}$} \\
\hline & & & & & & \multirow{2}{*}{$\begin{array}{c}\text { Alternative } \\
\tilde{z}\left(t_{\text {end }}\right) \\
(\mathrm{g})\end{array}$} & \multirow{2}{*}{$\begin{array}{c}\text { ROSESIM } \\
\hat{z}\left(t_{\text {beg }}\right) \\
(\mathrm{g})\end{array}$} & \\
\hline $\begin{array}{l}\text { Treatment } \\
\text { no. }\end{array}$ & $\begin{array}{c}\text { PPF } \\
\left(\mu \mathrm{mol} \cdot \mathrm{m}^{-2} \cdot \mathrm{s}^{-1}\right)\end{array}$ & $\begin{array}{l}\text { DT } \\
\left({ }^{\circ} \mathrm{C}\right)\end{array}$ & $\begin{array}{l}\mathrm{NT} \\
\left({ }^{\circ} \mathrm{C}\right)\end{array}$ & & & & & \\
\hline \multirow[t]{3}{*}{$\overline{6}$} & 175 & 20 & 17 & Low: & & 1.15 & 5.78 & 6.51 \\
\hline & & & & Mean & & 6.82 & 5.79 & \\
\hline & & & & Higl & & 24.94 & 5.85 & \\
\hline \multirow[t]{3}{*}{10} & 175 & 15 & 17 & Low: & & 3.94 & 7.44 & 8.74 \\
\hline & & & & Mean & & 11.95 & 7.45 & \\
\hline & & & & High: & & 85.65 & 7.52 & \\
\hline \multirow[t]{3}{*}{12} & 250 & 23 & 30 & Low: & & 0.508 & 5.02 & 5.96 \\
\hline & & & & Mean & & 7.67 & 5.07 & \\
\hline & & & & High: & & 11.04 & 5.10 & \\
\hline
\end{tabular}

${ }^{2} \mathrm{PPF}=$ photosynthetic photon flux; $\mathrm{DT}=$ day temperature; $\mathrm{NT}=$ night temperature.

y Treatment means as follows. Treatment numbers relate directly to those in Hopper et al. (1994). Treatment 6: mean total dry weight from 50 plants of treatments 6 and 17-19 (all repeats of experiment design center point); treatments 10 and 12: mean total dry weight of 10 flowering shoots.

will not later fully recover, this is generally not the case over the range of environmental factors we studied. Because favorable conditions prevailed during the initial growth phase (Hopper et al., 1994), those conditions probably did not have an excessive long-term effect on later growth. Thus, environmental conditions during the main growth phase should have the greatest effect on growth, with relatively little dependence on the initial phase, which justifies the use of the additive polynomial approach in the ROSESIM model as described by Hopper et al. (1994).

\section{CONCLUSIONS AND RECOMMENDATIONS}

The information we have presented covers a broad range of responses for 'Royalty' roses, both in the rate of development and in total plant dry matter at flowering. We believe that these data highlight some of the advantages of the ROSESIM approach to modeling and simulation of the growth of 'Royalty' roses relative to an alternative approach that uses log-transformed responses.

Even in situations where the initial condition for the main growth phase is known quite closely, we believe that ROSESIM will yield more accurate predictions of each selected plant growth characteristic throughout the main growth phase because ROSESIM is based on carefully verified response-surface models for daily growth increments of the target response, not the log-transformed response. In the alternative model of the main growth phase that we have discussed in this paper (and which was attempted in practice), the exponentiation op- eration required to recover the original (untransformed) response at flowering introduced a substantial bias into the final prediction; and this bias also affected the accuracy of the intermediate predictions within the main growth phase.

Cumulative distribution functions (cdfs) and inverse cdfs have many similarity to growth functions; in particular, the prediction bias mentioned in the last paragraph is similar to the estimator bias that arises when we estimate inverse cdfs by Hora's method (1983). Closely paralleling the operations required by the alternative growth model, Hora's method for estimating inverse cdfs involves the following operations on a sample data set: 1 ) applying a transformation defined by the logarithm of a certain function of the original data; 2) estimating an associated rate of change for the transformed response using a linear regression model; 3) accumulating the increments of the transformed response as predicted by the linear regression model; and 4) untransforming the final accumulated result by taking antilogarithms to obtain an estimate of the inverse cdf from which the original responses were sampled. Avramidis and Wilson (1994) explored the problems with Hora's method for estimating inverse cdfs, and the analysis given by Avramidis and Wilson also sheds some light on the causes of prediction bias in the alternative model of the main growth phase.

We should emphasize that the ROSESIM approach (Hopper et al., 1994) for modeling the growth of 'Royalty' roses differs fundamentally from the approach that underlies classical empirical growth models. ROSESIM attempts to model the daily growth increment as a direct function of the prevailing environmental conditions $X_{\mathrm{PPF}}, X_{\mathrm{DT}}$, and $X_{\mathrm{NT}}$ and the (coded) time $X_{\mathrm{t}}$ as shown in Eq. [6]. This method is a modification of the state-rate method as presented by de Wit and Goudriaan (1978), where (coded) time $X_{\mathrm{t}}$ acts as a state variable and the prevailing environmental conditions $X_{\mathrm{PPF}}, X_{\mathrm{DT}}$, and $X_{\mathrm{NT}}$ are the driving or forcing variables. ROSESIM provides a stepwise iterative solution (de Wit and Goudriaan, 1978) using Euler integration (Conte and de Boor, 1980) rather than a strictly analytical solution. By contrast, the classical approach attempts to model the growth rate, $d z(t) / d t$, as a function of $z(t)$ alone such that an analytical solution of the resulting differential equation can be readily obtained (Richards, 1969). For example, Richards' growth model (Richards, 1959) is based on the differential equation

$\frac{d z(t)}{d t}=k \cdot z(t) \cdot\left\{\frac{[A / z(t)]^{1-m}-1}{1-m}\right\}$

$(A, k, m \geq 0)$,

where 1) $A$ is the asymptotic maximum level (final size) of the response; 2) $k$ is the rate constant that determines the spread of the growth curve along the time axis; and 3) $m$ is a shape parameter that determines basic mathematical properties of the growth curve, including the relative growth rate, $[d z(t) / d t] / z(t)$, of the response over time and the proportion of the final size at which the inflection point of the growth curve occurs. Eq. [17] has the analytical solution

$z(t)=A \cdot\left[1-\theta \cdot e^{-k \bullet t}\right]^{1 /(1-m)}$

$(\theta<0$ if $m>1$; otherwise $\theta \geq 0)$

in which the auxiliary parameter $\theta$ determines the initial condition for the response. If a classical growth curve such as Eq. [18] were used to model rose growth in the context described in this paper, then in general each combination of the environmental factors $X_{\mathrm{PPF}}$, $X_{\mathrm{DT}}$, and $X_{\mathrm{NT}}$ would require a different set of parameter estimates for $A, k, m$, and $\theta$. Such an approach would be prohibitively cumbersome to implement and use. Moreover, it is unclear how Eqs. [17] and [18] could be adapted to time-varying values of $X_{\mathrm{PPF}}, X_{\mathrm{DT}}$, or $X_{\mathrm{NT}}$.

ROSESIM, however, achieves a more parsimonious representation of the daily growth increments as a single third-degree polynomial function of $X_{\mathrm{PPF}}, X_{\mathrm{DT}}, X_{\mathrm{NT}}$, and $X_{\mathrm{t}}$ having the form specified in Eq. [6]. The verification of ROSESIM presented in Hopper et al. (1994), together with the argument presented in this paper, provide evidence that ROSESIM constitutes a more flexible and comprehensive approach to modeling the growth of 'Royalty' roses under constant environmental conditions. We believe that the approach of ROSESIM can also be adapted to model the growth of other plants as a function of time and constant environmental factors, and that similar improvements can be achieved by using the additive polynomial approach of ROSESIM.

Although follow-up work is needed in a number of areas, perhaps the most immediate requirement is extension of the growth model 
described by Eqs. [1] through [9] to include random variation over time in uncontrollable (or partially controllable) environmental variable such as PPF. Although we have presented some preliminary results on an extension of ROSESIM to handle daily variations in natural solar radiation (Hopper et al., 1994), we have not performed a comprehensive validation of the prediction accuracy of this extended version of ROSESIM. For greenhouses lacking strict environmental control systems, some daily variation in DT and NT also will occur; and the extended version of ROSESIM must accurately represent dependence (correlation) between the environmental variables PPF, DT, and NT that are observed on successive days as well as the dependence of the latest observed plant growth characteristics on all previous observations of PPF, DT, and NT.
If such an extension of ROSESIM can be formulated, implemented, and validated, then ROSESIM might be integrated into schemes for 1) improved control of greenhouse environmental conditions and 2) more accurate prediction of crop maturity to coincide with major holidays.

\section{Literature Cited}

Avramidis, A.N. and J.R. Wilson. 1994. A flexible method for estimating inverse distribution functions in simulation experiments. ORSA J. Computing 6:342-355.

Conte, S.D. and C. de Boor. 1980. Elementary numerical analysis: An algorithmic approach. 3rd ed. McGraw-Hill, New York.

de Wit, C.T. and J. Goudriaan. 1978. Simulation of ecological processes. 2nd ed. Wiley, New York.

Draper, N.R. and H. Smith. 1981. Applied regression analysis. 2nd ed. Wiley, New York.
Hopper, D.A. and P.A. Hammer. 1991. Regression models describing Rosa hybrida L. response to day/night temperature and photosynthetic photon flux. J. Amer. Soc. Hort. Sci. 116:609-617.

Hopper, D.A., P.A. Hammer, and J.R. Wilson. 1994. A simulation model of Rosa hybrida growth response to constant irradiance and day and night temperatures. J. Amer. Soc. Hort. Sci. 119:903-914.

Hora, S.C. 1983. Estimation of the inverse function for random variate generation. Communications Assn. Computing Machinery 26:590-594.

Nelder, J.A. 1961. The fitting of a generalization of the logistic curve. Biometrics 17:89-110.

Richards, F.J. 1959. A flexible growth function for empirical use. J. Expt. Bot. 10:290-300.

Richards, F.J. 1969. The quantitative analysis of growth, p. 3-76. In: F.C Steward (ed.). Plant physiology: A treatise. vol. VA. An analysis of growth: Behavior of plants and their organs. Academic, New York.

\title{
INSERT REGENT INSTRUMENTS AD THIS IS A BLACK \& WHITE AD! (camera-ready copy enclosed)
}

\author{
DROP KEYLINE
}

